\title{
Biallelic MFSD2A variants associated with congenital microcephaly, developmental delay, and recognizable neuroimaging features
}

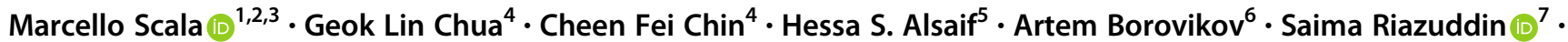 \\ Sheikh Riazuddin $^{8,9}$ - M. Chiara Manzini ${ }^{10}$ - Mariasavina Severino ${ }^{11}$ - Alvin Kuk ${ }^{4}$ Hao Fan ${ }^{12,13,14}$. \\ Yalda Jamshidi ${ }^{15}{ }^{15}$ Mehran Beiraghi Toosi ${ }^{16} \cdot$ Mohammad Doosti $^{16}$. Ehsan Ghayoor Karimiani ${ }^{16}$.

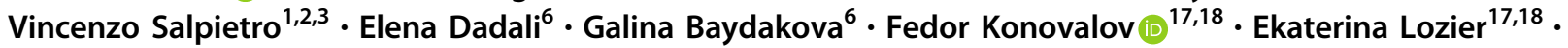 \\ Emer O'Connor $^{1}$ - Yasser Sabr $\mathbb{D}^{19}$ - Abdullah Alfaifi ${ }^{20}$ - Farah Ashrafzadeh ${ }^{21}$ - Pasquale Striano ${ }^{2,3}$.

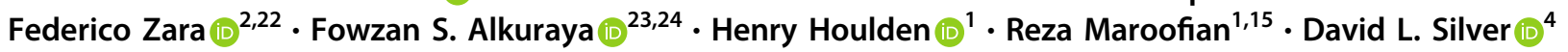

Received: 7 February 2020 / Revised: 30 April 2020 / Accepted: 4 June 2020 / Published online: 22 June 2020

(c) The Author(s), under exclusive licence to European Society of Human Genetics 2020

\begin{abstract}
Major Facilitator Superfamily Domain containing 2a (MFSD2A) is an essential endothelial lipid transporter at the blood-brain barrier. Biallelic variants affecting function in MFSD2A cause autosomal recessive primary microcephaly 15 (MCPH15, OMIM\# 616486). We sought to expand our knowledge of the phenotypic spectrum of MCPH15 and demonstrate the underlying mechanism of inactivation of the MFSD2A transporter. We carried out detailed analysis of the clinical and neuroradiological features of a series of 27 MCPH15 cases, including eight new individuals from seven unrelated families. Genetic investigation was performed through exome sequencing (ES). Structural insights on the human Mfsd2a model and invitro biochemical assays were used to investigate the functional impact of the identified variants. All patients had primary microcephaly and severe developmental delay. Brain MRI showed variable degrees of white matter reduction, ventricular enlargement, callosal hypodysgenesis, and pontine and vermian hypoplasia. ES led to the identification of six novel biallelic MFSD2A variants (NG_053084.1, NM_032793.5: c.556+1G>A, c.748G>T; p.(Val250Phe), c.750_753del; p.(Cys251SerfsTer3), c.977G >A; p.(Arg326His), c.1386_1435del; p.(Gln462HisfsTer17), and c.1478C >T; p.(Pro493Leu)) and two recurrent variants (NM_032793.5: c.593C >T; p.(Thr198Met) and c.476C $>$ T; p.(Thr159Met)). All these variants and the previously reported NM_032793.5: c.490C $>$ A; p.(Pro164Thr) resulted in either reduced MFSD2A expression and/or transport activity. Our study further delineates the phenotypic spectrum of MCPH15, refining its clinical and neuroradiological characterization and supporting that MFSD2A deficiency causes early prenatal brain developmental disruption. We also show that poor MFSD2A expression despite normal transporter activity is a relevant pathomechanism in MCPH15.
\end{abstract}

These authors contributed equally: Marcello Scala, Geok Lin Chua

Supplementary information The online version of this article (https:// doi.org/10.1038/s41431-020-0669-x) contains supplementary material, which is available to authorized users.

Reza Maroofian

r.maroofian@ucl.ac.uk

$\triangle$ David L. Silver

david.silver@duke-nus.edu.sg

Extended author information available on the last page of the article

\section{Introduction}

Major Facilitator Superfamily Domain containing 2a (MFSD2A) is a sodium-dependent lysophosphatidylcholine (LPC) transporter that is highly expressed at the endothelium of the blood-brain barrier (BBB) [1]. Omega-3 fatty acids and other mono- and polyunsaturated fatty acids conjugated as LPCs are transported by MFSD2A, which plays a pivotal role in the supply of omega-3 fatty acids to the brain [1]. The essential role of MFSD2A in regulating lipogenesis in the developing brain has been recently demonstrated using loss-of-function mouse models [2].

Five distinct homozygous loss-of-function MFSD2A variants have been reported in patients with neurodevelopmental abnormalities from seven consanguineous families. 
These patients showed developmental delay (DD), microcephaly, and neuroimaging abnormalities such as ventriculomegaly and hypoplasia of the corpus callosum, brainstem, and cerebellum. These observations underscored the fundamental role of LPC transport at the BBB for human brain development and clarified the structurefunction relationships in the MFSD2A-mediated transport mechanism [3-9].

In this study, we report seven new families with biallelic variants affecting function in MFSD2A, expanding the phenotype and defining the characteristic neuroimaging features of MFSD2A-related neurodevelopmental disorder, also known as Autosomal Recessive Microcephaly 15, (MCPH15, OMIM \#616486). We provide clinical, genetic, and functional characterization of these novel variants and the previously reported NM_032793.5:c.593C $>$ T; p.(Thr198Met) and c.490C $>$ A; p.(Pro164Thr) variants on the transporter activity, which further substantiates the functional importance of LPC transport for human brain development.

\section{Materials and methods}

\section{Patients ascertainment}

Eight patients from seven unrelated families were locally referred for exome sequencing (ES) in the context of severe microcephaly and psychomotor delay. Patients were enrolled in accordance with the Declaration of Helsinki and informed consent was obtained for all of them in agreement with the requirements of Iranian, Pakistani, Russian, and Saudi bioethics laws. Subjects were examined by several geneticists, neurologists, and pediatricians with expertise in pediatric neurology. Detailed family history was collected for all families. Brain MRIs were locally acquired with different protocols, but all included diffusion-weighted images, T1- and T2-weighted, and FLAIR images on the 3 planes. Images were reviewed by an experienced pediatric neuroradiologist (MS) and a pediatrician with expertise in neurogenetics (MS) in consensus. Blood samples were obtained from patients and parents.

\section{Exome sequencing}

After standard DNA extraction from peripheral blood, proband-only ES was performed in all the families as previously described [10-12]. Variants were filtered out according to frequency, conservation, and predicted impact on protein function by several bioinformatic tools (SIFT, Polyphen-2, Mutation Taster). Candidate variants were subsequently validated through co-segregation studies by Sanger sequencing and submitted to the gene variant database LOVD at https://databases.lovd.nl/shared/genes/ MFSD2A (Individual IDs 00276067, 00276070, 00276071, 00276074, 00276075, 00276076, 00276077). All the variants are reported according to the NM_032793.5 transcript. GeneMatcher was used for the distributed case-matching [13]. Further details available in the Supplementary Methods.

\section{Functional tests summary methods}

Site-directed mutagenesis was used to create the Mfsd2a variants NM_032793.5:c.1478C >T; p.(Pro493Leu), c.593C> T; p.(Thr198Met), c.490C $>$ A; p.(Pro164Thr), c.977G $>A$; p.(Arg326His), and c.748G $>\mathrm{T}$; p.(Val250Phe) in a mammalian expression vector, which were used to determine the effects on transporter function in mammalian cells. The amino acid variants in Mfsd2a protein were modeled and visualized to understand the causative mechanism of transporter dysfunction. Further details are available in the Supplementary Methods.

\section{Results}

\section{Clinical features}

We present eight patients (Table 1) from seven unrelated families of varying ancestry (Saudi, Iranian, Pakistani, and Russian), including six consanguineous families (Families A, B, C, E, F, and G) (Fig. 1a, b).

Patient 1 (Family A) is a 4-year-old female born to consanguineous parents (first-cousins) of Iranian ancestry. Prenatal ultrasound revealed microcephaly. At birth, her occipital frontal circumference (OFC) was $28 \mathrm{~cm}(-4.6 \mathrm{SDS})$. At the age of 6 months, she had head-lag, was unable to roll over, and lacked babbling. At 1 year of age, she started to suffer from myoclonic seizures and failure to thrive (FTT) due to dysphagia. Physical examination at 4 years showed progressive microcephaly with an OFC of $41 \mathrm{~cm}(-5.6 \mathrm{SDS})$ and bilateral talipes equinovarus (TEV). She was unable to walk and neurological examination revealed spastic quadriparesis and hyperreflexia. Karyotyping and metabolic testing were normal.

Patient 2 (Family B) is 4-year-old Iranian male born to consanguineous parents. Family history revealed several previous miscarriages. His older brother was healthy. At birth, his OFC was $27 \mathrm{~cm}$ (-3.9 SDS). He was diagnosed with global DD during infancy and started to suffer from generalized tonic-clonic seizures since the age of 2 years. At 4 years, he was unable to sit and his language was very limited. Physical examination revealed bilateral TEV, progressive microcephaly with OFC of $37 \mathrm{~cm}(-8.8 \mathrm{SDS})$ and spastic quadriparesis. 


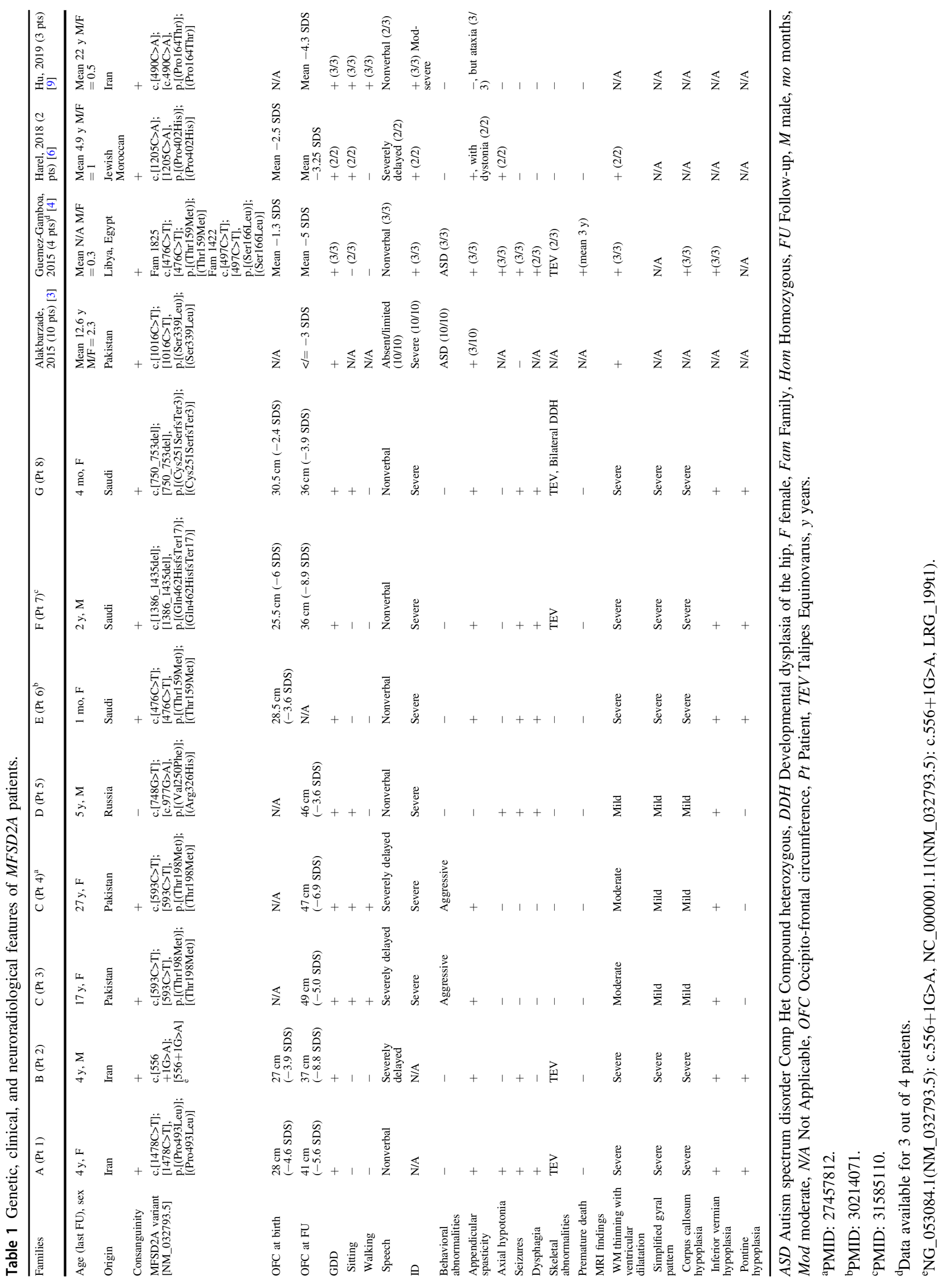


a

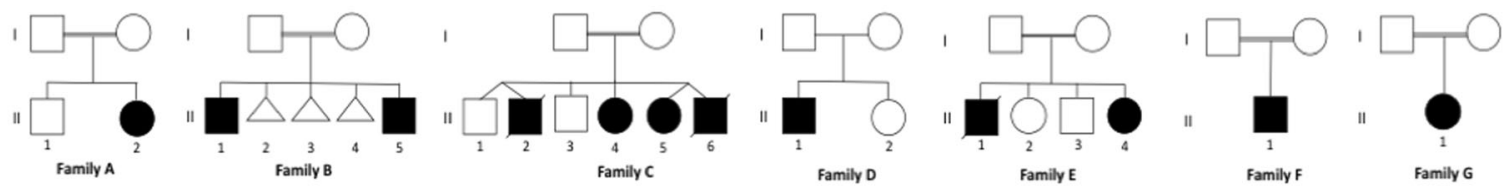

b

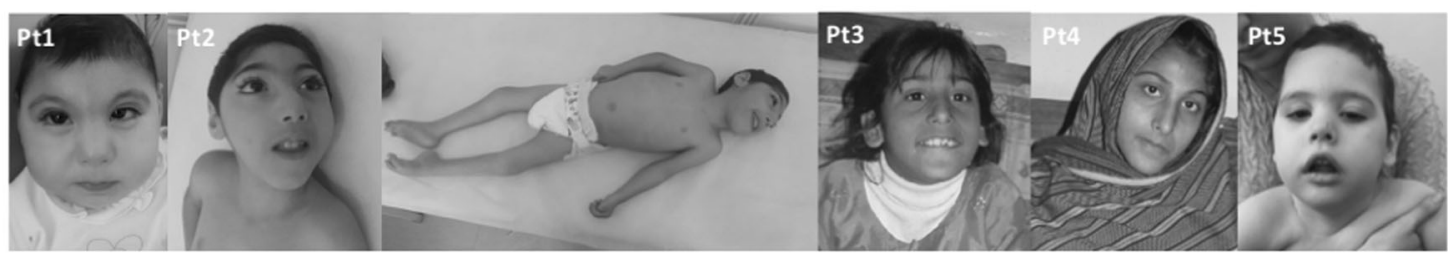

c

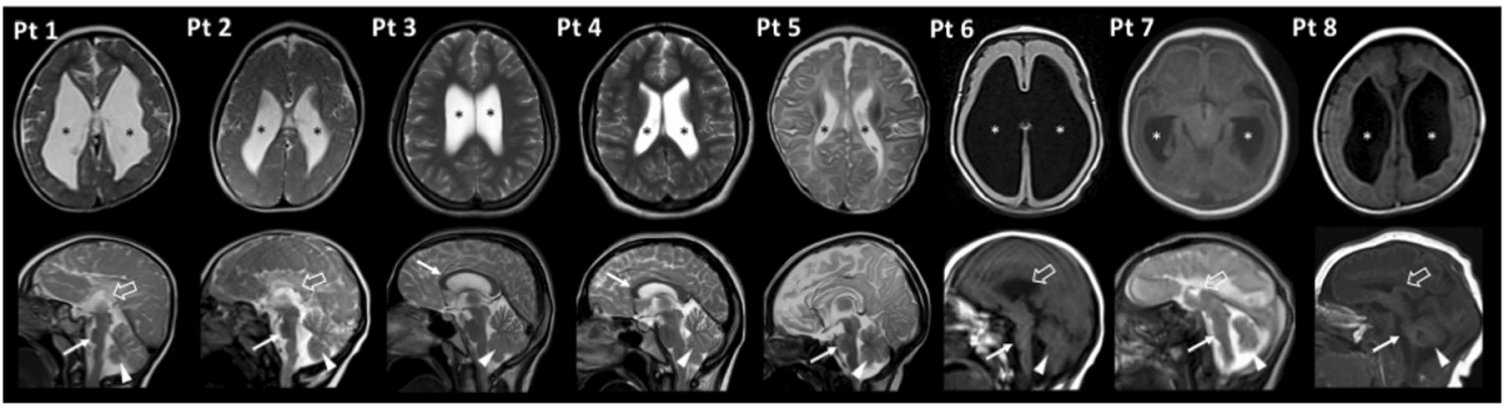

d

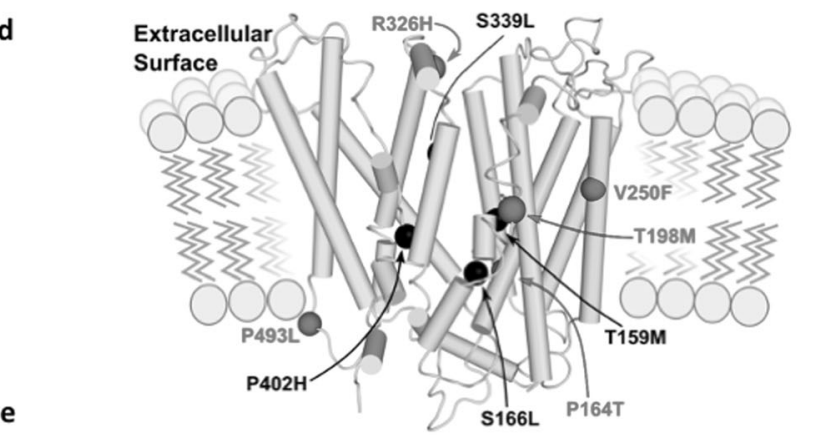

e
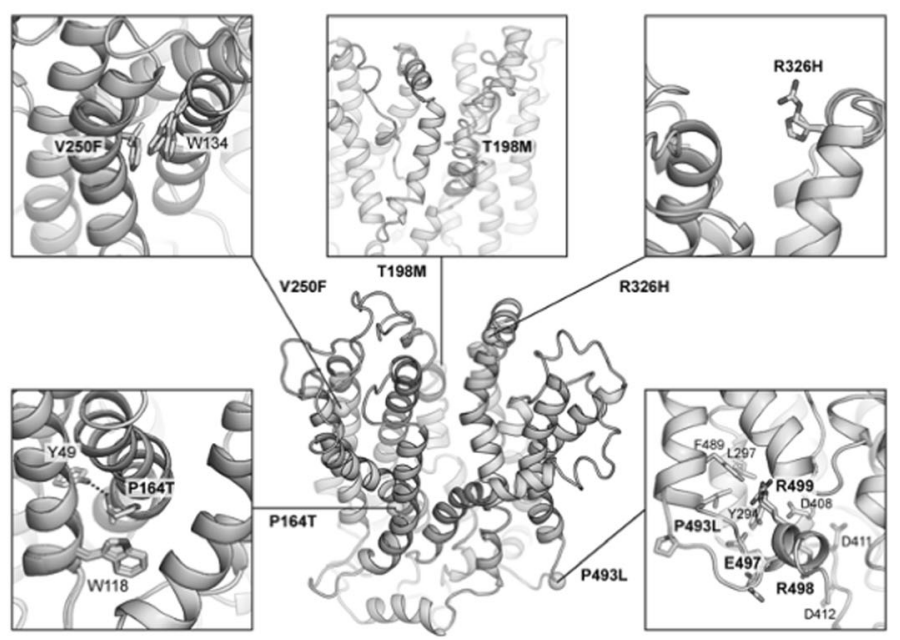

f
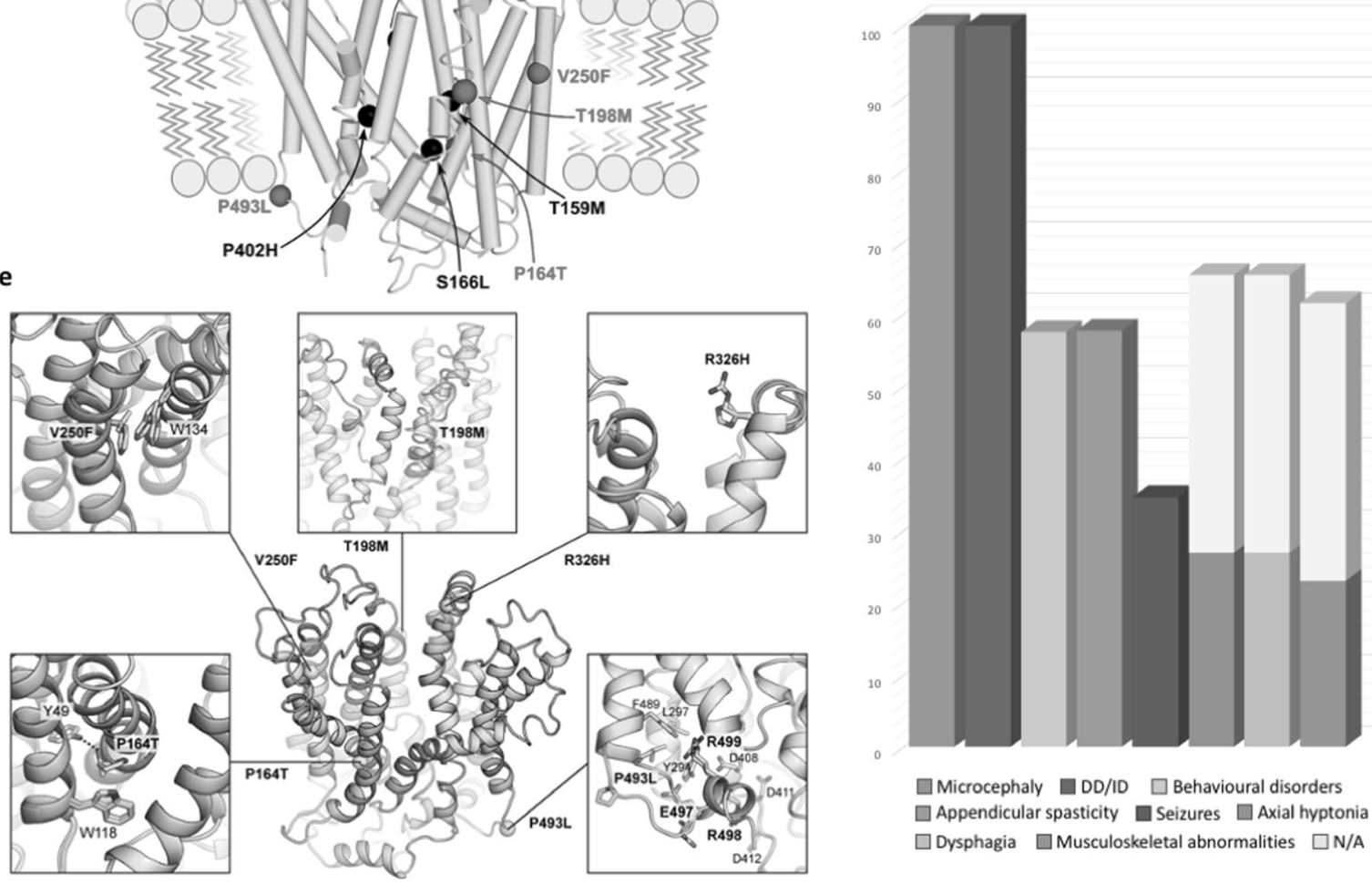

Patient 3 and 4 (Family C) belong to a consanguineous family of Pakistani descent consisting of six siblings. Two males were reported to have microcephaly and died in the neonatal period due to a possible infection. Two males were healthy. The proband (patient 3), a 17-year-old female, and her sister (patient 4), currently 27 years old, presented with 
Fig. 1 Clinical characterization, neuroimaging features, genetic findings and predicted consequences of MFSD2A variants. a Pedigrees of the seven reported families. b Main clinical features include severe microcephaly, axial hypotonia, talipes equinovarus, and minor dysmorphic features (e.g., epicanthal folds and broad nasal bridge in patient 5). c Brain MRI of affected subjects performed at 3 years (Pt 1), 1 year (Pt 2), 17 years (Pt 3), 27 years (Pt 4), 2 months (Pt 5), 1 month (Pt 6), 2 years (Pt 7), and 4 months of age (Pt 8). First row: axial T2, FLAIR or T1-weighted images of the patients. Second row: corresponding sagittal T2- or T1-weighted images. There is severe microcephaly with mildly to severely simplified gyral pattern in all subjects. The cerebral white matter is reduced with consequent ventricular dilatation (asterisks), especially in patients 1, 2, 6, 7, and 8 . The corpus callosum is barely visible and markedly short in patients 1 , 2, 6, 7, and 8 (empty arrows), while it is diffusely hypoplastic in Patient 5. Hypoplasia of the anterior portion of the corpus callosum is visible in patients 3 and 4 (arrows). Note that in all subjects the cingulate gyrus is present. The inferior portion of the vermis is small in all subjects (arrowheads), with associated pontine hypoplasia in patients 1, 2, 5, 6, 7, and 8. d 3D structural model of Mfsd2a (based on Quek DQ et al. 2016; Supplementary References) indicating the locations of previously reported variants (in black) and the variants identified in this study (in red). The N-terminus is indicated in green and C-terminus in cyan. e 3D structural models of the Mfsd2a variants. Positions of variants in the human Mfsd2a protein. Variants (cyan) were mapped to the published homology model of Mfsd2a (green). R326 is located at the putative extracellular gate and the R326H substitution might disrupt gate closure. V250 and P164 are both located in helical bundles. Their substitution by larger amino acids (V250F and P164T) might perturb protein folding by steric clash with neighboring sidechains (e.g., W134, W118). P164T might also form a hydrogen bond with Y49 that is not seen in canonical Mfsd2a. Variants T198M and P493L are predicted to alter the local protein structure. f Percentage distribution of the main clinical features of $M F S D 2 A$ patients. $D D$ developmental delay; $I D$ intellectual disability; N/A not applicable; $P t$ patient.

severe global DD and aggressive behavior during infancy. They had no seizure history. Physical evaluation revealed mild muscle weakness, language limited to few words, and severe microcephaly, with an OFC of $49 \mathrm{~cm}$ ( -5.0 SDS) and $47 \mathrm{~cm}$ ( -6.9 SDS) in patients 3 and 4, respectively.

Patient 5 (Family D) is the youngest of two siblings born to unrelated parents of Russian descent. Neonatal history was unremarkable except for microcephaly. The baby started to suffer from generalized tonic-clonic seizures at the age of 1 month. Global DD was subsequently diagnosed at 1 year of age as he was unable to sit without support and could not speak. At 5 years, the patient was unable to walk and nonverbal. He had microcephaly with OFC of $46 \mathrm{~cm}$ (-3.6 SDS), gross and fine motor impairment, and axial hypotonia. He also had dysphagia, excessive drooling, and some dysmorphic features, including wide nasal bridge and prominent epicanthal folds.

Patient 6 (Family E) is a 1-month-old Saudi female born to consanguineous parents. She was the youngest of four siblings. Her older brother had microcephaly but died during infancy. The patient was diagnosed with severe microcephaly at birth, with an OFC of $28.5 \mathrm{~cm}$ (-6.2 SDS).
During the neonatal period she suffered from FTT due to severe dysphagia and physical examination further revealed generalized spasticity.

Patient 7 (Family F) is a 2-year-old male born to consanguineous parents from Saudi Arabia. During the neonatal period, he suffered from FTT and received percutaneous endoscopic gastrostomy (PEG) due to severe dysphagia. At 1 year of age, he started to suffer from recurrent seizures treated with phenobarbital and sodium valproate. Developmental milestones were severely delayed. The patient was also diagnosed with gastro-esophageal reflux. Physical examination showed microcephaly, bilateral TEV, generalized muscle weakness, and spasticity.

Patient 8 (Family G) is a 4-month-old female born to consanguineous Saudi parents. Prenatal ultrasound showed microcephaly and fetal echogenic bowel. Perinatal course was uneventful, but at the age of 1 week the baby was admitted to neonatal intensive care unit due to relevant feeding difficulties. At 4 months, she started to suffer from seizures requiring hospitalization. Physical examination showed microcephaly, generalized spasticity, bilateral hip dislocation, and left TEV.

\section{Neuroimaging}

Brain MRI revealed mild to severe white matter reduction with consequent ventricular dilatation in all subjects (Fig. 1c). In particular, the supratentorial white matter was markedly thinned with severe ventriculomegaly in $5 / 8$ patients. The degree of myelination was appropriate for the age in all subjects. The cortical gyral pattern was mildly to severely simplified in all cases, without other associated cortical malformations. The thalami were small and the corpus callosum was abnormal in all patients. In particular, in 5 subjects the corpus callosum was markedly thin and short, in 2 patients there was hypoplasia of the anterior portion of the corpus callosum, while in the remaining patient it was globally thin. Of note, the cingulate gyrus was present in all subjects. Finally, inferior vermian hypoplasia was observed in all cases, while pontine hypoplasia was present in 6/8 patients.

\section{Genetic findings}

After filtering for allele frequency, conservation, and predicted functional impact, biallelic MFSD2A variants were prioritized as candidate disease-causing variants. Eight different variants were identified (Fig. 1d), including three homozygous missense variants (c.1478C $>$ T; p.(Pro493Leu) in patient 1; c.593C >T; p.(Thr198Met) in patient 3 and 4; c.476C $>\mathrm{T}$; p.(Thr159Met) in patient 6), a homozygous splice site variant (patient 2: NG_053084.1(NM_032793.5): c.556+1G>A, NC_000001.11(NM_032793.5): c.556+1G> A, LRG_199t1), two homozygous frameshift variants 
(c.1386_1435del; p.(Gln462HisfsTer17) in patient 7; c.750_753del; p.(Cys251SerfsTer3) in patient 8), and two compound heterozygous missense variants (c.[748G $>\mathrm{T}]$; [977G $>$ A $]$, p.[(Val250Phe $)] ;[($ Arg326His $)]$ in patient 5) (Table 2). Biparental segregation confirmed the autosomal recessive inheritance model. In Family $\mathrm{C}$ (Fig. 1a), unaffected individuals (II-1 and II-3) were heterozygous for the c.593C > T; p.(Thr198Met) variant in MFSD2A, whereas the DNA of the deceased individuals (II-2 and II-6) was not available due to their premature death. All the identified variants are absent in the homozygous state and extremely rare in the heterozygous state in the most common population databases (including our database of 10,000 exomes, gnomAD, Greater Middle East Variome - GME, Iranome, and Ensembl). Missense variants were located at the amino acid residues with high levels of conservation, with a Genomic Evolutionary Rate Profiling (GERP) score between 5.49 to 5.94. The predicted effect on protein function was also consistent with a loss-of-function mechanism, with a Combined Annotation Dependent Depletion (CADD) score ranging from 24.4 to 34 . The two frameshift variants are predicted to result in nonsense-mediated mRNA decay, likely leading to a functional knock-out. All the identified variants are predicted to be damaging by several bioinformatic tools, such as SIFT, Polyphen-2, and Mutation Taster. The splicing variant c.556+1G $>\mathrm{A}$ is predicted to result in aberrant splicing through the alteration of the wildtype (WT) donor site by Human Splice Finder and Variant Effect Predictor.

\section{Mfsd2a variants lead to loss-of-function and/or loss- of-expression}

Human Mfsd2a is a 530 amino acid glycosylated sodiumdependent MFS transporter composed of 12 conserved transmembrane domains [7]. To understand the consequence of the c.1478C $>\mathrm{T}$; p.(Pro493Leu), c.490C $>\mathrm{A}$; p.(Pro164Thr), c.593C $>$ T; p.(Thr198Met), c.977G $>$ A; p.(Arg326His), and c.748G $>\mathrm{T}$; p.(Val250Phe) variants on the structure and function of $\mathrm{Mfsd} 2 \mathrm{a}$, we utilized a published structural model of human Mfsd2a to carry out bioinformatic predictions [7]. In the c.593C $>\mathrm{T}$; p.(Thr198Met) mutant model, M198 faces the internal cavity of the transporter and forms more favorable hydrophobic interactions with neighboring residues such as F399 from helix X, in comparison to T198 in the WT model that faces the membrane exterior (Fig. 1e). In the c.1478C $>\mathrm{T}$; p.(Pro493Leu) mutant model, the proline-to-leucine amino acid change results in the extension of helix XII that is stabilized by a hydrophobic cluster formed by sidechains of L493 and three other residues Y294, L297, and F489 (Fig. 1e). In addition, multiple polar interactions observed in the WT model are absent in the c.1478C $>\mathrm{T}$;
p.(Pro493Leu) mutant model, including the hydrogen bonding interaction between Y294 and E497 as well as ionic locks between R498 and a negatively charged surface comprising D408, D411, and D412. These ionic locks were previously suggested to be important for the transporter function [7]. Taken together, we observed enhanced hydrophobic packing in both mutant models likely leading to increased structure rigidity and reduced mobility of the transporter, indirectly inactivating the transport of substrate. Additionally, the c.1478C >T; p.(Pro493Leu) mutant would be predicted to show a reduction in transport due to the partial loss of ionic locks.

We next utilized HEK293 cells, which do not endogenously express Mfsd2a, as an in vitro cell system to determine if $\mathrm{Mfsd} 2 \mathrm{a}$ variants affect protein expression, localization, and transport function. Mock transfected and the sodium binding transporter inactive mutant p.(Asp97Ala) (p.(D97A)) served as negative controls [1, 7], while WT Mfsd2a served as a positive control. Western blot analysis of WT Mfsd2a showed the multiple protein bands similar to results previously reported for overexpression of Mfsd2a in HEK293 cells [3, 4, 6], while all the five mutants c.1478C $>$ T; p.(Pro493Leu), c.593C $>$ T; p.(Thr198Met), c.490C $>$ A; p.(Pro164Thr), c.977G >A; p.(Arg326His), and c.748G >T; p.(Val250Phe) were expressed at less than $30 \%$ of WT Mfsd2a (Fig. 2a). This low level of protein expression of these five Mfsd2a mutants is consistent with predicted negative effects of these variants on protein folding (Fig. 1e). Despite low level expression of all five Mfsd2a mutants, immunofluorescence microscopy indicated that all mutants were expressed at the plasma membrane similarly to WT (Fig. 2b).

To directly test the functional consequences of these five variants on LPC transport, we utilized an established transport assay that quantifies net transport of ${ }^{14} \mathrm{C}-\mathrm{LPC}$ DHA in HEK293 cells. To directly compare transport activity between WT and the five mutants c.1478C $>\mathrm{T}$; p.(Pro493Leu), c.593C $>$ T; p.(Thr198Met), c.490C $>$ A; p.(Pro164Thr), c.977G >A; p.(Arg326His), and c.748G >T; p.(Val250Phe), we first titrated down the amount of plasmid DNA for the transfection of WT Mfsd2a into cells to obtain a comparable expression level of WT to all five mutants. We found that $0.1 \mu \mathrm{g}$ of WT yielded similarly low levels of expression as cells transfected with $2 \mu \mathrm{g}$ of mutants (Fig. 2c). Surprisingly, at comparable protein expression levels of WT and mutants, four of the five mutants demonstrated comparable transport of 14C-LPC-DHA in HEK293 cells with c.593C>T; p.(Thr198Met) at $75 \%$, c.490C $>\mathrm{A}$; p.(Pro164Thr) at $82 \%$, c.977G $>\mathrm{A}$; p.(Arg326His) at $104 \%$, and c.748G $>\mathrm{T}$; p.(Val250Phe) at $80 \%$ of WT transport activity. Only P493L was similar to non-functional D97A negative control, indicating it is inactive (Fig. 2d). 


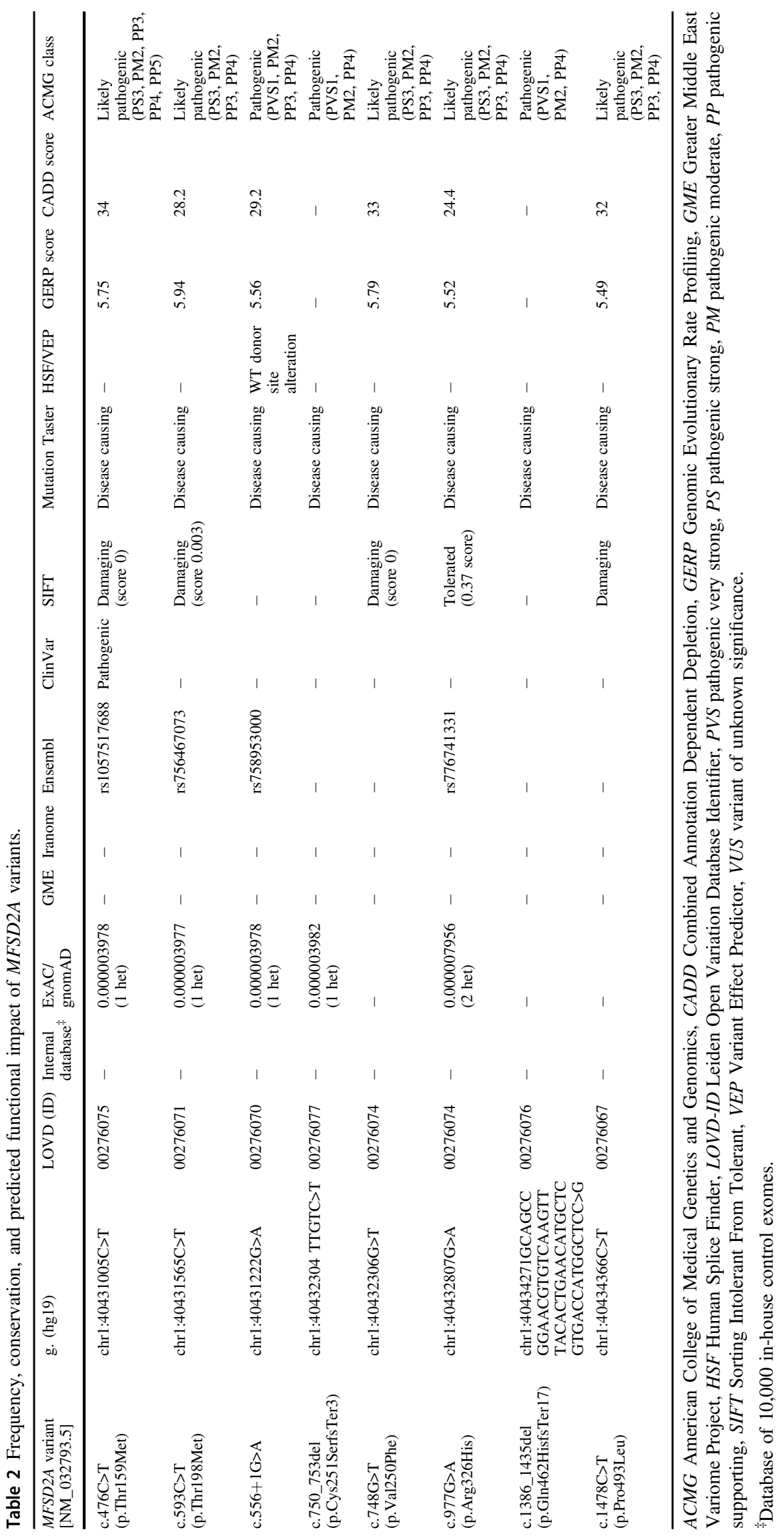


Fig. 2 Biochemical analysis of Mfsd2a variants. a Western blot probed for Mfsd2a and its mutants with $\beta$-actin used as loading control. b Confocal immunofluorescence micrographs of transiently transfected HEK293 cells with Mock, WT, D97A, P493L, T198M, P164T, R326H and V250F variants affecting function showing Mfsd2a localization in green cell nuclei in blue (Hoechst stain), red arrows pointing to the cell surface localization of Mfsd2a and its mutants. c Titration of varying amounts of WT Mfsd2a DNA $(\mu \mathrm{g})$ to normalize the expression levels to determine the amount of WT Mfsd2a needed for comparable expression levels with cells transfected with $2 \mathrm{mg}$ of mutant construct DNA. d Transport of $50 \mu \mathrm{M}{ }^{14} \mathrm{C}$ LPC-DHA by comparable expression levels of MFSD2A in HEK293.

Significance levels of difference compared with the transport activity of $0.1 \mu \mathrm{g}$ of WT Mfsd2a (labeled WT on the graph).

Transport activity are labeled with asterisks: $* * * *$ representing $P$ value $<0.0001, * * *$ representing $P$ value $<0.001$, ** representing $P$ value $<0.01$, * representing $P$ value $<0.1$. a

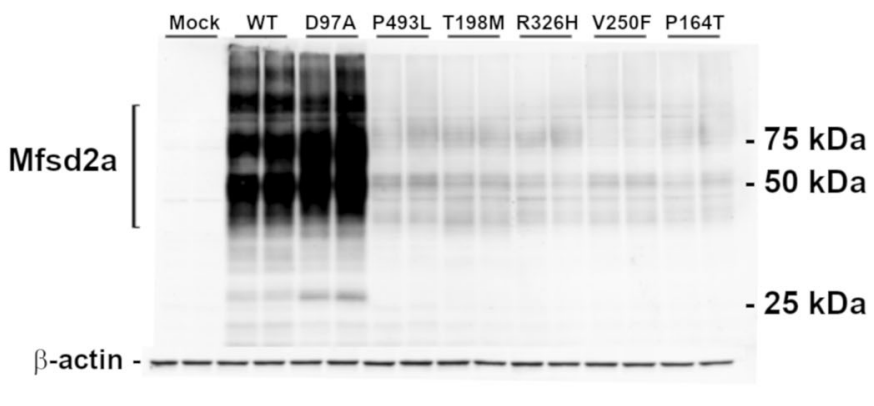

b
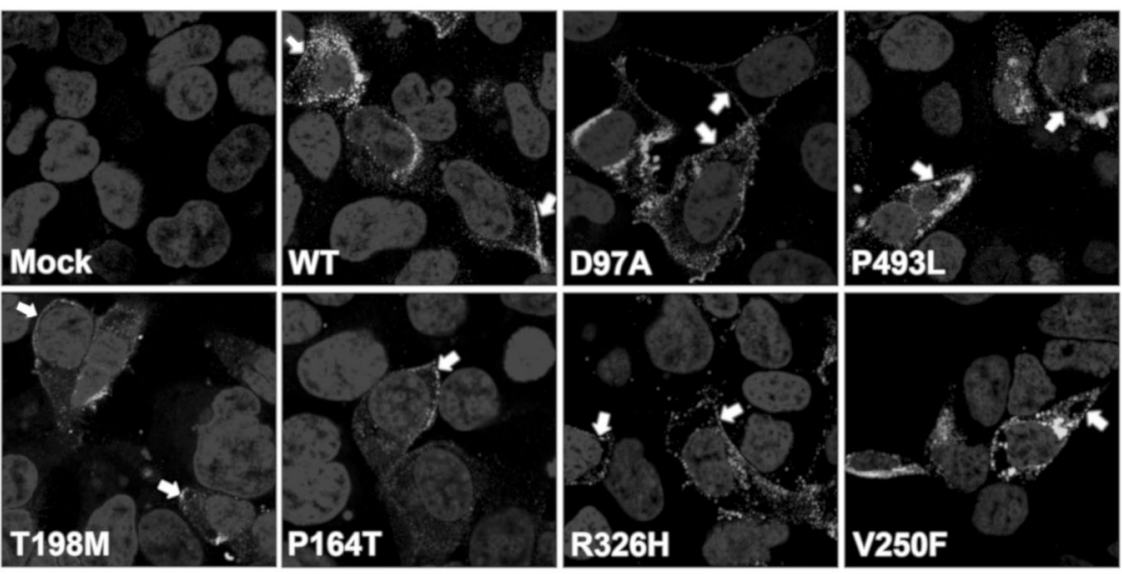

c
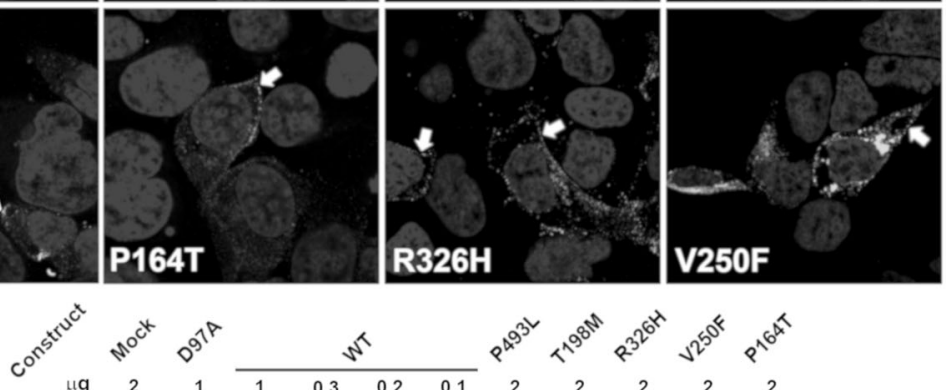

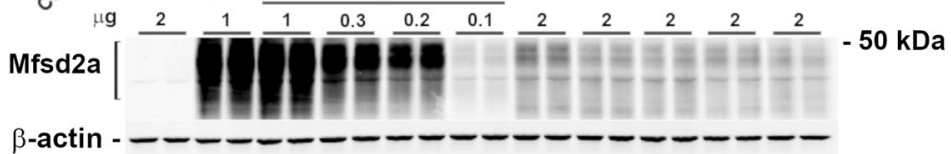

d

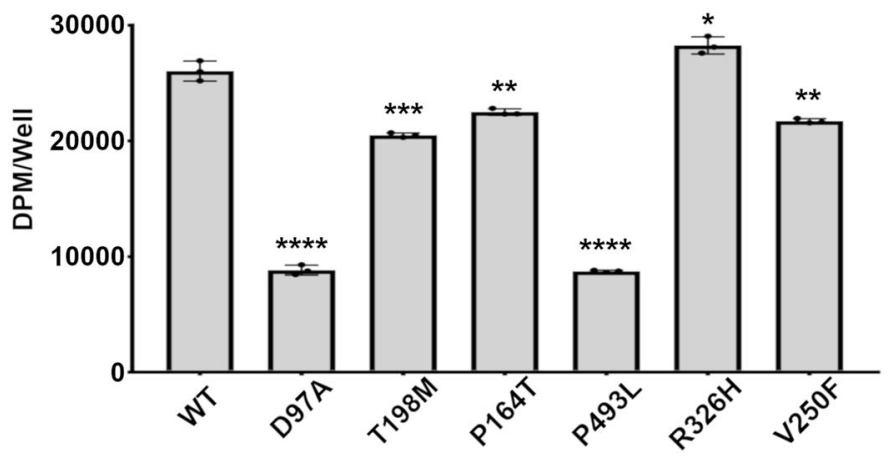

Previously reported non-synonymous variants in Mfsd2a have been shown to affect transport function but not protein expression $[3,4,6]$. In our cases, five of the variants (c.593C $>\mathrm{T}$; p. (Thr198Met), c.490C $>\mathrm{A}$; p.(Pro164Thr), c.977G $>$ A; p.(Arg326His), c.748G $>\mathrm{T}$; p.(Val250Phe), and c.1478C $>$ T; p.(Pro493Leu)) were extremely lowly expressed (Fig. 2a). Our findings indicate that poor expression of Mfsd2a, despite normal transporter activity, can also be an underlying cause for severe microcephaly and hypomyelination in these patients, which further defines the etiology of Mfsd2a-related microcephaly.

\section{Discussion}

MFSD2A is a sodium-dependent 12-pass transmembrane protein belonging to the major facilitator superfamily of secondary transporters. Mfsd2a plays a pivotal role at the BBB for the transport of plasma-derived LPCs conjugated to polyunsaturated fatty acids such as the omega-3 fatty acid docosahexaenoic acid (DHA) to the brain $[1,2,14]$. The deficiency of the DHA in the brain of $M f s d 2 a$-knockout mice is associated with a severe neurodevelopmental phenotype characterized by microcephaly, cognitive impairment, ataxia, and severe anxiety [12]. In particular, 
microcephaly is likely explained by the fact that LPC transport not only provides accretion of DHA by the developing brain, but is also critical for providing LPC as building blocks for neuron arborization and regulation of membrane phospholipid composition $[2,5,15]$. The reports of loss-of-function MFSD2A variants in patients with a progressive microcephaly syndrome with severe ID and neuroimaging abnormalities have supported the relevant role of this lipid transporter in human brain development and functioning [3, 4, 9]. The relevance of proper DHA metabolism for brain development and functioning is further supported by CYP2U1 deficiency. This enzyme is a member of the cytochrome $\mathrm{P} 450$ family 2 subfamily $U$ and catalyzes the hydroxylation of arachidonic acid (AA) and AA-related long-chain fatty acids, including DHA [16]. Biallelic loss-of-function CYP2U1 variants cause spastic paraplegia 56 (SPG56), a complex neurological condition characterized by spasticity, cognitive impairment, and white matter abnormalities [16].

Here, we present seven families with eight distinct lossof-function variants in MFSD2A, including seven novel variants affecting function. Patient 4 was part of a large cohort of consanguineous families with recessive intellectual disability reported by Riazuddin et al [8]. Patients 6 and 7 were briefly described before by Shaheen et al. and Monies et al., respectively $[17,18]$. In line with previously reported cases, our patients showed a complex neurodevelopmental phenotype primarily characterized by severe progressive microcephaly, ID, spasticity, and speech delay (Table 1) (Fig. 1f) [3, 4, 6, 8, 9]. Less common clinical features were also identified in our cohort, including axial hypotonia, increased deep tendon reflexes, and seizures (Fig. 1b) [3, 4, 6, 8, 9]. Of note, none of our patients died prematurely, although some of their siblings who died prematurely were most likely affected by the same condition. The longest follow-up was 27 years (patient 4), allowing assessment of the progression of microcephaly over time. Language was delayed in most subjects and one patient was nonverbal. Four patients showed skeletal abnormalities consistent with TEV. Dysmorphic features were observed in patient 5 only.

In previously reported cases, brain MRI revealed a spectrum of abnormal findings, including ventricular enlargement secondary to white matter paucity and hypoplasia of the corpus callosum, cerebellum, and brainstem [3, 4]. In our study, we provide further evidence that affected subjects present severe microcephaly with simplified gyral pattern, associated with variable degrees of white matter reduction leading to mild to severe ventricular dilatation. Of note, the myelination was always appropriate for patients' age in our series, ruling out a hypomyelinating disorder. Interestingly, the corpus callosum was always abnormal, with severe hypodysplasia in most subjects. However, the cingulate gyrus was present in the most severe cases as well, indicating that the corpus callosum was initially formed. Finally, the inferior cerebellar vermis was small in all subjects while hypoplasia of the pons was noted in almost all of them. Taken together, these neuroimaging features are consistent with an early prenatal developmental disruption and likely suggest a relevant role of LPCs in the development of both the cerebral gray and white matter.

A clear correlation between the severity of the clinicoradiological phenotype and the variants affecting function in MFSD2A could not be observed. Despite the MFSD2A variants identified in the current study impair protein expression rather than the transporter function, no substantial difference between the phenotypes of previously reported affected individuals and patients from the current cohort was noticed (Table 1). This observation supports the loss of function as the main pathogenic mechanism in MCPH15, regardless of the specific underlying cause. All patients show a variable degree of progressive microcephaly and a comparable level of psychomotor delay, but some speculations on selected phenotypic features are possible. In fact, behavioral disturbances appeared to be more frequent in subjects carrying missense variants affecting the transporter function (c.1016C $>\mathrm{T}$; p.(Ser339Leu), c.476C >T; p.(Thr159Met), and c.497C >T; p.(Ser166Leu)) $[3,4]$, whereas skeletal abnormalities might be more common in patients carrying variants resulting in decreased MFSD2A expression, as showed by patients $1,2,7$, and 8 from our cohort. Interestingly, extrapyramidal disorders have been associated with the previously reported variants c.1205C >A; p.(Pro402His) and c.490C $>$ A; p.(Pro164Thr) $[6,9]$, but were absent in our cases. As to the neuroimaging features, the degree of involvement of gray and white matter structures is quite variable in the affected individuals and does not appear to be correlated to MFSD2A variant type.

In conclusion, our observations expand the phenotypic spectrum of MFSD2A-related microcephaly syndrome and provide new insights into the underlying pathogenic mechanisms. Refining the neuroradiological characterization of MCPH15, we suggest that some neuroimaging clues can be extremely relevant for an early diagnosis. We also show that poor MFSD2A expression plays a relevant role in MCPH15 pathogenesis, further defining the etiology of this condition. A better understanding of the role of MFSD2A in brain physiology will foster the development of targeted therapies or specific metabolic supplementation regimens to bypass LPC transport deficiency. The identification and characterization of further patients harboring loss-of-function MFSD2A variants will support efforts to exploit LPCs as therapeutic lipids to improve DHA delivery and promote proper brain development in affected individuals. 
Acknowledgements The work was supported in part by National Research Foundation and Ministry of Health grants, Singapore; by the Biomedical Research Council of A*STAR; by March of Dimes Research Grant; as part of the Queen Square Genomics group at University College London, supported by the National Institute for Health Research University College London Hospitals Biomedical Research Centre.

Funding National Research Foundation grants, Singapore NRF2016 NRF-NRFI001-15 and OF-IRG MOH-000217 (to DLS); Biomedical Research Council of A*STAR (to HF); The MRC (MR/S01165X/1, MR/S005021/1, G0601943), The National Institute for Health Research University College London Hospitals Biomedical Research Centre, Rosetree Trust, Ataxia UK, MSA Trust, Brain Research UK, Sparks GOSH Charity, Muscular Dystrophy UK (MDUK), Muscular Dystrophy Association (MDA USA), March of Dimes USA (to MCM), The R01 RNS107428A by the National Institute of Neurological Disorders and Stroke/National Institutes of Health (NINDS/NIH).

\section{Compliance with ethical standards}

Conflict of interest The authors declare that they have no conflict of interest.

Publisher's note Springer Nature remains neutral with regard to jurisdictional claims in published maps and institutional affiliations.

\section{References}

1. Nguyen LN, Ma D, Shui G, Wong P, Cazenave-Gassiot A, Zhang $\mathrm{X}$, et al. Mfsd2a is a transporter for the essential omega-3 fatty acid docosahexaenoic acid. Nature. 2014;509:503-6.

2. Chan JP, Wong BH, Chin CF, Galam DLA, Foo JC, Wong LC, et al. The lysolipid transporter Mfsd2a regulates lipogenesis in the developing brain. PLoS Biol. 2018;16:e2006443.

3. Alakbarzade V, Hameed A, Quek DQ, Chioza BA, Baple EL, Cazenave-Gassiot A, et al. A partially inactivating mutation in the sodium-dependent lysophosphatidylcholine transporter MFSD2A causes a non-lethal microcephaly syndrome. Nat Genet. 2015;47: 814-7.

4. Guemez-Gamboa A, Nguyen LN, Yang H, Zaki MS, Kara M, Ben-Omran T, et al. Inactivating mutations in MFSD2A, required for omega-3 fatty acid transport in brain, causea lethal microcephaly syndrome. Nat Genet. 2015;47:809-13.
5. Guesnet P, Alessandri JM. Docosahexaenoic acid (DHA) and the developing central nervous system (CNS) - implications for dietary recommendations. Biochimie. 2011;93:7-12.

6. Harel T, Quek DQY, Wong BH, Cazenave-Gassiot A, Wenk MR, Fan $\mathrm{H}$, et al. Homozygous mutation in MFSD2A, encoding a lysolipid transporter for docosahexanoic acid, is associated with microcephaly and hypomyelination. Neurogenetics. 2018;19:227-35.

7. Quek DQ, Nguyen LN, Fan H, Silver DL. Structural insights into the transport mechanism of the human sodium-dependent lysophosphatidylcholine transporter Mfsd2a. J Biol Chem. 2016;291: 9383-94.

8. Riazuddin S, Hussain M, Razzaq A, Iqbal Z, Shahzad M, Pollaet DL, et al. Exome sequencing of Pakistani consanguineous families identifies 30 novel candidate genes for recessive intellectual disability. Mol psychiatry. 2017;22:1604-14.

9. Hu H, Kahrizi K, Musante L, Fattahi Z, Herwig R, Hosseini M, et al. Genetics of intellectual disability in consanguineous families. Mol Psychiatry. 2019;24:1027-39.

10. Li H, Durbin R. Fast and accurate short read alignment with Burrows-Wheeler transform. Bioinformatics. 2009;25:1754-60.

11. Van der Auwera GA, Carneiro MO, Hartl C, Poplin R, Del Angel G, Levy-Moonshine A, et al. From FastQ data to high confidence variant calls: the Genome Analysis Toolkit best practices pipeline. Curr Protoc Bioinforma. 2013;43:11.10.1-11.10.33.

12. Wang $\mathrm{K}, \mathrm{Li}$ M, Hakonarson H. ANNOVAR: functional annotation of genetic variants from high-throughput sequencing data. Nucleic Acids Res. 2010;38:e164.

13. Sobreira N, Schiettecatte F, Valle D, Hamosh A. GeneMatcher: a matching tool for connecting investigators with an interest in the same gene. Hum Mutat. 2015;36:928-30.

14. Andreone BJ, Chow BW, Tata A, Lacoste B, Ben-Zvi A, Bullock $\mathrm{K}$, et al. Blood-brain barrier permeability is regulated by lipid transport-dependent suppression of caveolae-mediated transcytosis. Neuron. 2017;94:581-94.

15. Ahmad A, Moriguchi T, Salem N. Decrease in neuron size in docosahexaenoic acid deficient brain. Pediatr Neurol. 2002;26:210-8.

16. Tesson C, Nawara M, Salih MA, Rossignol R, Zaki MS, Al Balwi $\mathrm{M}$, et al. Alteration of fatty-acid-metabolizing enzymes affects mitochondrial form and function in hereditary spastic paraplegia. Am J Hum Genet. 2012;91:1051-64.

17. Shaheen R, Maddirevula S, Ewida N, Alsahli S, Abdel-Salam GMH, Zaki MS, et al. Genomic and phenotypic delineation of congenital microcephaly. Genet Med. 2019;21:545-52.

18. Monies D, Abouelhoda M, Assoum M, Moghrabi N, Rafiullah R, Almontashiri N, et al. Lessons learned from large-scale, first-tier clinical exome sequencing in a highly consanguineous population. Am J Hum Genet. 2019;104:1182-201.

\section{Affiliations}

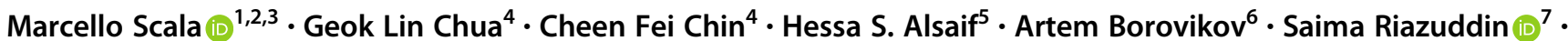
Sheikh Riazuddin ${ }^{8,9} \cdot$ M. Chiara Manzini ${ }^{10}$ - Mariasavina Severino ${ }^{11}$ - Alvin Kuk ${ }^{4}$ Hao Fan ${ }^{12,13,14}$. Yalda Jamshidi $\mathbb{D}^{15}$. Mehran Beiraghi Toosi ${ }^{16}$. Mohammad Doosti ${ }^{16}$. Ehsan Ghayoor Karimiani ${ }^{16}$. Vincenzo Salpietro $^{1,2,3}$. Elena Dadali ${ }^{6}$. Galina Baydakova ${ }^{6}$. Fedor Konovalov $\mathbb{D}^{17,18}$ - Ekaterina Lozier ${ }^{17,18}$. Emer $\mathrm{O}^{\prime}$ Connor $^{1} \cdot$ Yasser Sabr $\mathbb{D}^{19}{ }^{19}$ Abdullah Alfaifi $^{20}$ - Farah Ashrafzadeh ${ }^{21}$ - Pasquale Striano ${ }^{2,3}$. Federico Zara $\mathbb{D}^{2,22} \cdot$ Fowzan S. Alkuraya $\mathbb{D}^{23,24} \cdot$ Henry Houlden $\mathbb{1}^{1} \cdot$ Reza Maroofian ${ }^{1,15} \cdot$ David L. Silver $\left(\mathbb{D}^{4}\right.$

1 Department of Neuromuscular Disorders, Institute of Neurology, University College London, London, UK
2 Department of Neurosciences, Rehabilitation, Ophthalmology, Genetics, Maternal and Child Health, University of Genoa, Genoa, Italy 
3 Pediatric Neurology and Muscular Diseases Unit, IRCCS Istituto Giannina Gaslini, Genoa, Italy

4 Signature Research Program in Cardiovascular and Metabolic Disorders, Duke-NUS Medical School, Singapore 169857, Singapore

5 Department of Genetics, King Faisal Specialist Hospital and Research Centre, Riyadh, Saudi Arabia

6 Research Centre for Medical Genetics, Moscow, Russia

7 Department of Otorhinolaryngology Head \& Neck Surgery, School of Medicine, University of Maryland, Baltimore, MD 21201, USA

8 Center for Genetic Diseases, Shaheed Zulfiqar Ali Bhutto Medical University, Pakistan Institute of Medical Sciences, Islamabad, Pakistan

9 National Centre of Excellence in Molecular Biology, University of the Punjab, Lahore 53700, Pakistan

10 Department of Neuroscience and Cell Biology and Child Health Institute of New Jersey, Rutgers Robert Wood Johnson Medical School, New Brunswick, NJ 08901, USA

11 Neuroradiology Unit, IRCCS Istituto Giannina Gaslini, Genoa, Italy

12 Bioinformatics Institute, Agency for Science, Technology and Research (A*STAR), 30 Biopolis St., Matrix No. 07-01, Singapore 138671, Singapore
13 Department of Biological Sciences, National University of Singapore, 14 Science Drive 4, Singapore 117543, Singapore

14 Centre for Computational Biology, DUKE-NUS Medical School, 8 College Road, Singapore 169857, Singapore

15 Genetics Research Centre, Molecular and Clinical Sciences Institute, St George's, University of London, Cranmer Terrace, London SW17 ORE, UK

16 Department of pediatric diseases, Faculty of medicine, Mashhad University of Medical Sciences, Mashhad, Iran

17 Independent Clinical Bioinformatics Laboratory, Moscow, Russia

18 Genomed Ltd., Moscow, Russia

19 Department of Obstetrics and Gynecology, King Saudi University, Riyadh, Saudi Arabia

20 Pediatrics Department, Security Forces Hospital, Riyadh, Saudi Arabia

21 Department of Pediatric Diseases, Mashhad University of Medical Sciences, Mashhad, Iran

22 Unit of Medical Genetics, IRCCS Istituto Giannina Gaslini, Genova, Italy

23 Department of Genetics, King Faisal Specialist Hospital and Research Center, Riyadh, Saudi Arabia

24 Department of Anatomy and Cell Biology, College of Medicine, Alfaisal University, Riyadh, Saudi Arabia 